\title{
Color-Accurate Underwater Imaging Using Perceptual Adaptive Illumination
}

\author{
Iuliu Vasilescu, Carrick Detweiler, and Daniela Rus \\ Distributed Robotics Laboratory, CSAIL \\ Massachusetts Institute of Technology \\ Cambridge, MA 02139 \\ Email: iuliuv@tiaresearch.com, \{carrick, rus\}@csail.mit.edu
}

\begin{abstract}
Capturing color in water is challenging due to the heavy non-uniform attenuation of light in water across the visible spectrum, which results in dramatic hue shifts toward blue. Yet observing color in water is important for monitoring and surveillance as well as marine biology studies related to species identification, individual and group behavior, and ecosystem health and activity monitoring. Underwater robots are equipped with motor control for large scale transects but they lack sensors that enable capturing color-accurate underwater images. We present a method for color-accurate imaging in water called perceptual adaptive illumination. This method dynamically mixes the illumination of an object in a distance-dependent way using a controllable multi-color light source. The color mix compensates correctly for color loss and results in an image whose color composition is equivalent to rendering the object in air. Experiments were conducted with a color palette in the pool and at three different coral reefs sites, and with an underwater robot collecting image data with the new sensor.
\end{abstract}

\section{INTRODUCTION}

Underwater monitoring of natural as well as humanengineered environments can be greatly enhanced by automated capture of underwater color images using underwater robots $[8,13]$. Our goal is to develop color-accurate automated imaging of underwater environments using robots capable of capturing, mosaicing, mapping, and analyzing color images of the space. In our previous work [12] we have described a robot capable of such a task. Figure 7 shows this underwater robot at the end of an underwater color imaging mission. In this paper we discuss an algorithm and instrument that enables robots to image objects such as coral heads and wrecks by automatically collecting a succession of underwater color-accurate images. Color encodes important information and plays a key role in underwater object identification, monitoring and surveillance, and marine biology — for example a healthy sea anemone is pigmented by zooxenthella, while an unhealthy sea anemone is bleached.

Current cameras are not able to capture color in water at distances greater than $1 \mathrm{~m}$, which makes color-dependent underwater studies challenging [6]. Part of the challenge for underwater imaging is that color is unavailable as an accurate data parameter for distances greater than $1 \mathrm{~m}$ due to the uneven attenuation of the color spectrum as light travels through water. Longer wavelengths (e.g., red light) attenuate more rapidly than shorter wavelengths (e.g., blue light), resulting in dramatic hue shifts toward blue. Absorption,
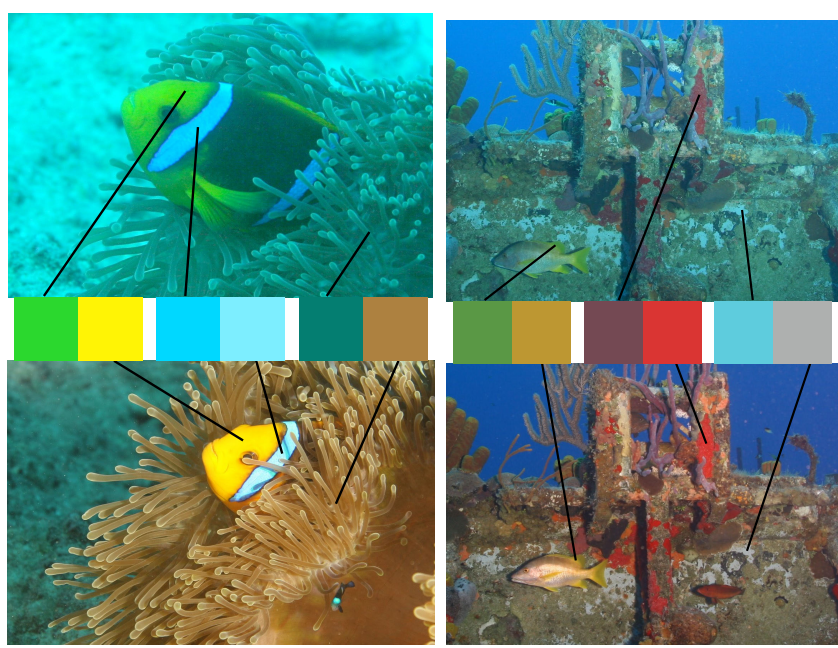

Fig. 1. Comparison of underwater imaging using white flash and adaptive illumination in a coral reef setting and a wreck setting. The color row in the middle shows color patches extracted from these images for visualization without the scene context. The left image shows a clown-fish and sea anemone in Moorea with white flash (Top) and adaptive illumination (Bottom). The pictures were taken at $20 \mathrm{~m}$ depth by Melissa Holbrook Schmitt. The scene was at a distance of $3 \mathrm{~m}$. The right image shows a coral formation on a ship wreck in the Grand Cayman with existing white flash (Top) and adaptive illumination (Bottom). The pictures were taken at $15 \mathrm{~m}$ depth by Cathy Church. The scene was at a distance of $3 \mathrm{~m}$.

the primary cause for color loss in water, is exponential with respect to the propagation distance. The exponential varies according to the wavelength of the light [9]. Other factors such as scattering contribute to image loss but are substantially independent of the wavelength of the visible light and do not affect color. Current approaches to underwater imaging rely on flooding objects with white light from a very close distance (e.g. less than $0.5 \mathrm{~m}$ ), using color filters, and doing manual post-processing. These techniques are cumbersome, do not render accurate colors, and work only for fixed setups, as color loss is distance-dependent. For example, Figure 1(Top) shows underwater images taken with current techniques at $3 \mathrm{~m}$ distance.

In this paper we describe a new method for color-accurate imaging in water called perceptual adaptive illumination. Our method uses knowledge about the physical processes that lead to the color shift. It computes light loss given distance 
to the imaged object and compensates for light loss using an illuminant whose radiation spectrum is controlled to be approximately the inverse of the water transfer function. The human color perception models are used for computing the optimal radiation spectrum. The full dynamic range of the camera is used and post-processing is not necessary. The end result is the color-accurate presentation of the object's image to the camera's CCD. Color-accurate imaging renders the object as if the image was taken in air, without the color loss side effects of water.

Figure 1 shows the comparison between images taken in water, using white flash and using perceptual adaptive illumination. The top pictures were taken with a white Xenon flash. The bottom figures were taken using the perceptual adaptive illumination method described in this paper. The color row in between the pictures shows color patches extracted manually from the images, presented without the scene context.

Underwater imaging with perceptual adaptive illumination requires a controllable illumination source capable of estimating distances. We have developed an instrument, algorithm, and software for capturing color-accurate images underwater by adaptive illumination which includes a spectrumcontrollable light source. The light composition is calculated so that this composition is transformed into white light by the water between the camera and the subject. The energy content of the light source is calculated using the optical properties of water and the distance to the subject. The light is generated by a source composed of several filtered Xenon light bulbs. Varying the relative power of the Xenon flashes effectively results in a light source with variable spectrum. Intuitively, the device senses the distance of the object and mixes the light of a multi-color flash so as to compensate for each component of the color spectrum according to known physics of how that particular light frequency dissipates in water. Distance sensing is accomplished with an acoustic sonar, by using the distance information from the camera's auto-focus system or can be manually entered by the operator.

We present data from a suite of pool experiments and ocean experiments using our system. We use a color palette and the $\mathrm{L}^{*} \mathrm{a} * \mathrm{~b} *$ color metric for evaluating color accuracy. Our experiments demonstrate color-accurate imaging in one image plane at distances ranging from $1 \mathrm{~m}$ to $5 \mathrm{~m}$ and at depths ranging from $5 \mathrm{~m}$ to $30 \mathrm{~m}$. Distance to the imaged object was measured in three different ways: using a measuring tape, using an external distance sensor, and using auto-focus information from the camera. We have attached the imaging apparatus to our underwater robot (see Figure 7(Right)) and used it to collect underwater images. We do not report in detail on the underwater robot missions because the focus of the paper is the underwater imaging system.

\section{A. Related Work}

Color plays a very important role in underwater monitoring and surveillance, as well as in marine biology studies related to species and behavior identification [2]. Many recognized species of coral reef fishes exhibit two or more color variants
[7]. A recent study [1] found that fish use color to communicate.

Current approaches to color imaging underwater rely on flooding objects with white light from close distances (e.g. less than $0.5 \mathrm{~m}[3]$ ), possibly followed by post-processing $[15,11]$. The post-processing step approximates the color of the image by manually setting a white point and correcting the image uniformly so that the selected point appears white. Since the color attenuation is not uniform this technique will not render accurate colors. These methods address the blue color shift by making assumptions about the properties of the picture (e.g. tagging known white patches in the picture). They do not use any knowledge of the physical process that led to color shift.

When objects of known color are present in the picture, various statistical or learning approaches have been applied [11]. Another option is to add a color filter to the camera to block much of the blue light, correcting for the loss of red [3]. Since absorption is distance-dependent, a different filter is needed for each distance. All these current techniques are cumbersome, do not render accurate colors, and produce color approximations for fixed distances and configurations only. What is needed is a method for adaptively illuminating an underwater scene with the correct light mix for each distance and depth. This will achieve color-accurate images without post processing. Accurate coloring in water refers to the equivalence between the color spectrum that reaches the imaging device in water to the color spectrum for the same object in air.

A recent paper by Yamashita et al. [15] considers the problem from a fundamental perspective and models the water effects on the color using the wavelength-dependent absorption coefficients and distance between camera and subject. The computed inverse function is applied to the captured image. The function is only a very coarse approximation that takes into account only the effect on three particular light wavelengths instead of the continuous spectrum. This simplification limits the performance and is not scalable to significant distances.

\section{B. Outline}

This paper is organized as follows. Section II introduces the physics of color perception in water. Section III discusses color perception by the human visual system and its implications to computation. Section IV presents the adaptive illumination algorithm. Section V describes the adaptive illumination instrument. Finally, Section VI presents our experimental data and evaluation.

\section{Color PERCEPTION IN WATER}

In this section we discuss the physics of color perception in water and the intuition behind how knowledge about colorabsorption in water can be used to do color-accurate imaging in water.

There are two important phenomena that affect imaging in water: scattering and absorption. Scattering is the physical process whereby light is forced to deviate from straight trajectory 


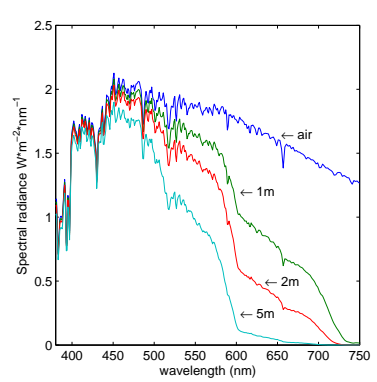

Fig. 2. The simulated spectrum of sunlight after it travels through $1 \mathrm{~m}, 2 \mathrm{~m}$, and $5 \mathrm{~m}$ of water. The simulation uses published sun spectrum and published light attenuation coefficients of water [9].

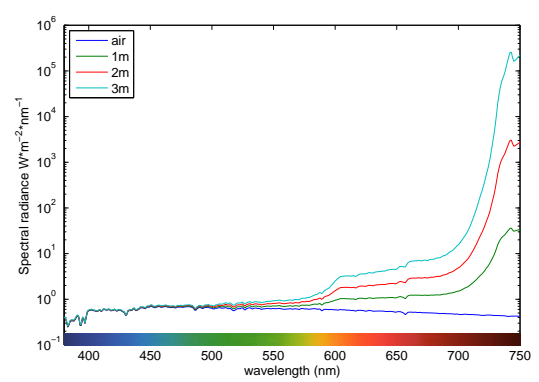

Fig. 3. The light required to compensate for color absorption in water. This is the inverse of water light transfer function plotted in Figure 2. The data is presented on a logarithmic scale due to its high dynamic range.

as it travels through water. The effect is caused predominantly by solid particles suspended in water, but also by the water molecules and substances dissolved in water. Scattering is wavelength independent and does not affect the color balance of the underwater image. This paper is not concerned with scattering.

Absorption is caused by water molecules and dissolved substances which convert light energy into heat. The water absorption coefficient, $a_{w}$, is wavelength dependent. Within the visible spectrum, longer wavelengths are attenuated stronger than shorter wavelengths (see Figure 2). Absorption is responsible for the color shift in underwater imaging. The absorption law describing the light energy transmitted by water is exponential.

$$
I(\lambda, d)=I(\lambda, 0) e^{-a_{w}(\lambda) d}
$$

where:

$\lambda$

$$
\text { light wavelength }
$$

$I(\lambda, 0) \quad$ spectral power distribution at source

$a_{w}(\lambda) \quad$ water absorption coefficient

$I(\lambda, d) \quad$ spectral power distribution at distance $d$ from the source, in water

The distance light travels through water (and is attenuated) is double the distance between subject and camera $d_{s c}$.

One solution is to compensate exactly for light absorption (see Figure 3). For example, given the camera to subject distance, the $650 \mathrm{~nm}$ red light is attenuated 5 times more than the $530 \mathrm{~nm}$ green light; thus, the light source should output 5 times more power at $650 \mathrm{~nm}$ than at $530 \mathrm{~nm}$. In general the spectral output power of the light source should be:

$$
I_{e c}\left(\lambda, d_{s c}\right)=\frac{I_{D 65}(\lambda)}{e^{-2 a_{w}(\lambda) d_{s c}}}=I_{D 65}(\lambda) e^{2 a_{w}(\lambda) d_{s c}}
$$

Such a light source compensates exactly for the light loss at the specified camera to subject distance $d_{s c}$. The subject appears as though illuminated by mid-day Sun light (D65 illuminant in air).

The usefulness of the brute-force method that relies on $I_{e c}\left(\lambda, d_{s c}\right)$ is very limited in practice. Fabricating a light source with the required spectral power distribution is challenging as the amount of optical power needed rises very sharply with distance (e.g. at $3 m$ the power required to take the picture in water is over $10^{5}$ times the power required to take the picture in air). In addition, existing light sources generally have fixed output spectra, which implies the use of filters to generate the required spectral distribution. This increases the power requirements by another order of magnitude.

Alternatively, we can exploit the scope of human color vision to save power.

\section{Color Perception by the Human Visual System}

A light source that can compensate exactly for light loss in water yields color-accurate pictures (at least for relatively planar scenes, where the distance between the subject and the camera or light source is relatively constant). However, this method is both wasteful in terms of power and resources, and not necessary as the human vision system is not a "perfect" light sampler. This section discusses the physics of the color vision system of humans. We use the properties of this system to create a low complexity and power efficient light source for color-accurate underwater imaging.

Creating the exact inverse of water attenuation will concentrate the majority of the energy in the deep red part of the spectrum where the water attenuation is the highest. However, it turns out that the human eye sensitivity is very low in this part of the spectrum. Very little extra accuracy would be achieved by exactly compensating for the deep red attenuation.

Exact compensation is not necessary since the eyes (and cameras) sample the spectrum with only three discrete color sensors, collapsing the infinite-dimensional color space into a three dimensional space. To preserve the human-perceived color accuracy, it is only necessary to preserve the color coordinates for naturally occurring colors in the three dimensional color space of humans. This allows for some tolerance in the light source spectrum.

Under typical conditions the objects around us are illuminated by a white light source $I_{0}(\lambda)$, which contains energy at all visible spectrum wavelengths. The color of an object is given by its wavelength-dependent reflection coefficient $R(\lambda)$ which represent the fraction of incident power that is reflected. Our eyes receive the reflected light as shown in Figure 4. The spectral distribution of the reflected light is given by the equation: $E(\lambda)=R(\lambda) I_{0}(\lambda)$. 


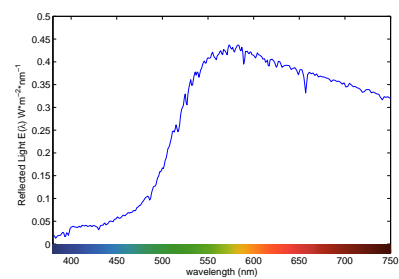

Fig. 4. Reflected spectral power of red color sample when lighted by a standard light (CIE illuminant D65).

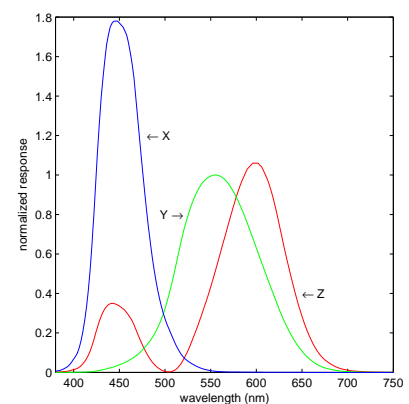

Fig. 5. The human visual system color response as modeled by the CIE $19312^{\circ}$ Standard Observer [4]. The 3 curves model the response of the three types of cone cell in human retina.

The human vision system perceives colors by sampling the light spectrum with three different types of cone cells $(\rho, \gamma$ and $\beta)$. Each type of cell is sensitive to a different region of the visible spectrum - long, medium and short wavelengths. The three normalized sensitivity curves $S_{\rho}(\lambda), S_{\gamma}(\lambda)$ and $S_{\beta}(\lambda)$ are plotted in Figure 6. The responses of these three types of cells $T_{\rho}, T_{\gamma}$ and $T_{\beta}$ are used by the central nervous system to associate colors to objects. For example, in the case of the Munsell 5Y8/10 color sample, illuminated with white light the responses of the three types of cone cells can be calculated as:

$$
\begin{gathered}
T_{\rho}^{5 Y 8 / 10}=\int_{380 \mathrm{~nm}}^{750 \mathrm{~nm}} S_{\rho}(\lambda) E(\lambda) d \lambda= \\
\int_{380 \mathrm{~nm}}^{750 \mathrm{~nm}} S_{\rho}(\lambda) R_{5 Y 8 / 10}(\lambda) I_{0}(\lambda) d \lambda \\
T_{\gamma}^{5 Y 8 / 10}=\int_{380 \mathrm{~nm}}^{750 \mathrm{~nm}} S_{\gamma}(\lambda) E(\lambda) d \lambda= \\
\int_{380 \mathrm{~nm}}^{750 \mathrm{~nm}} S_{\gamma}(\lambda) R_{5 Y 8 / 10}(\lambda) I_{0}(\lambda) d \lambda \\
T_{\beta}^{5 Y 8 / 10}=\int_{380 \mathrm{~nm}}^{750 \mathrm{~nm}} S_{\beta}(\lambda) E(\lambda) d \lambda= \\
\int_{380 \mathrm{~nm}}^{750 \mathrm{~nm}} S_{\beta}(\lambda) R_{5 Y 8 / 10}(\lambda) I_{0}(\lambda) d \lambda
\end{gathered}
$$

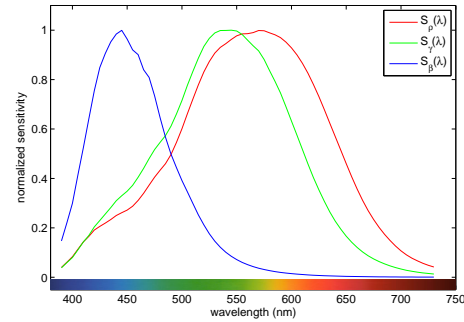

Fig. 6. Normalized sensitivities of the three types of cone cells present in human retina $(\rho, \gamma$ and $\beta)[10]$.

The brain receives a tuple of three numbers $\left(T_{\rho}, T_{\gamma}, T_{\beta}\right)$ and associates colors to objects based on this tuple. This results in an apparent three dimensional color space for humans. Any two objects that generate the same respective response in the three types of cone cells will appear to human observers as having the same color. This property can be exploited to reduce the complexity and power requirements of the underwater light source.

Using this observation, we develop a light source that preserves the coordinates of most naturally occurring colors in the human three dimensional color space, when used underwater. With this approach, the coordinates are also preserved in the color space of current cameras, since they closely model the human vision system color space.

Artificial light sources do not have the same spectral composition as the sun light. Therefore, they do not render the color of objects in the same way sunlight does. The Color Rendering Index (CRI) was developed by Commission Internationale de l'Éclairage (CIE) as a metric for comparing ability of light sources to render color accurately. The metric is based on comparing the color coordinates of 15 sample colors illuminated with the reference light and the tested light. The color samples have been chosen as representative for naturally occurring colors. The Euclidean distance between the color coordinates of two colors represented in CIE 1964 $U^{*} V^{*} W^{*}$ color space [14] is used as a quantitative value for color difference. $U^{*} V^{*} W^{*}$ is a uniform color space, meaning the Euclidean distance is a consistent measure of perceptual color difference, across the entire color space.

Our algorithm selects the power levels of a multi-color flash to maximize the CRI for objects illuminated in water. Formally, CRI is defined as:

$$
\begin{gathered}
\mathrm{CRI}=100-4.6 \Delta \bar{E}_{U * V * W *} \\
\Delta \bar{E}_{U * V * W *}=\frac{1}{15} \sum_{i=1}^{15} \Delta E_{i} \\
\Delta E_{i}=\sqrt{\Delta U_{i}^{2}+\Delta V_{i}^{2}+\Delta W_{i}^{2}}
\end{gathered}
$$

CRI has values between $0 \%$ and $100 \%$. A CRI of $100 \%$ corresponds to a perfect light source which renders colors identically to the reference light source. 


\section{Perceptual Adaptive Illumination}

Perceptual adaptive illumination is a power-efficient method for color-accurate imaging underwater. Given the distance to the object, the wavelength-dependent attenuation is computed using the known optical properties of water [9]. The light source spectrum is adjusted to compensate for this attenuation. The required light spectra is generated by a variable spectrum light source composed of several basic light sources, with fixed but distinct spectral power distributions. The variable spectrum is achieved by varying the relative power of the composing light sources. The spectral power distribution is optimized such that when filtered by the water between the camera and the subject, it will render the subject's human-perceived colors in the same way that natural light would render it in air. The optimization is performed by maximizing the apparent CRI of the light source when filtered by the water.

Given the camera-subject distance $d_{s c}$, adaptive illumination computes the optimal spectrum that can be generated by a source composed of $n$ light sources with fixed output spectra $I_{i}(\lambda)$ and independently adjustable output power $p_{i}$. The total output spectrum is a linear combination of the composing light sources:

$$
I(\lambda)=\sum_{i=1}^{n} p_{i} I_{i}(\lambda)
$$

The spectra of the component light sources can be viewed as the basis functions that are used to generate the required spectral power distribution. The scaling factors, $p_{i}$, correspond to the output power setting of the component light sources.

The generated light is filtered by the water between the light source and the subject, and also by water as it travels back from the subject to the camera. Thus, the total travel distance is $2 d_{s c}$, where $d_{s c}$ is the distance between subject and camera. Water attenuation makes the illumination equivalent to a light source in air:

$$
\hat{I}(\lambda)=e^{-2 a_{w}(\lambda) d_{s c}} I(\lambda)=e^{-2 a_{w}(\lambda) d_{s c}} \sum_{i=1}^{n} p_{i} I_{i}(\lambda)
$$

From the camera (and observer) perspective, the subject looks as though it is illuminated by $\hat{I}(\lambda)$ in air. The problem can be posed as adjusting $\hat{I}(\lambda)$ so that the colors are rendered accurately in the observer's color space.

The power settings are found by solving the following optimization problem for the subject to camera distance parameter $d_{s c}$ :

$$
\begin{gathered}
p_{1} . . p_{n}=\underset{p_{1} . p_{n} \in[0 . .1]}{\arg \max } \operatorname{CRI}(\hat{I}(\lambda))= \\
\underset{p_{1} . . p_{n} \in[0 . .1]}{\arg \max } \operatorname{CRI}\left(e^{-2 a_{w}(\lambda) d_{s c}} \sum_{i=1}^{n} p_{i} I_{i}(\lambda)\right)
\end{gathered}
$$

This optimization problem can be solved using any existing numerical optimization software. Algorithm 1 summarizes the computation for the optimal light.
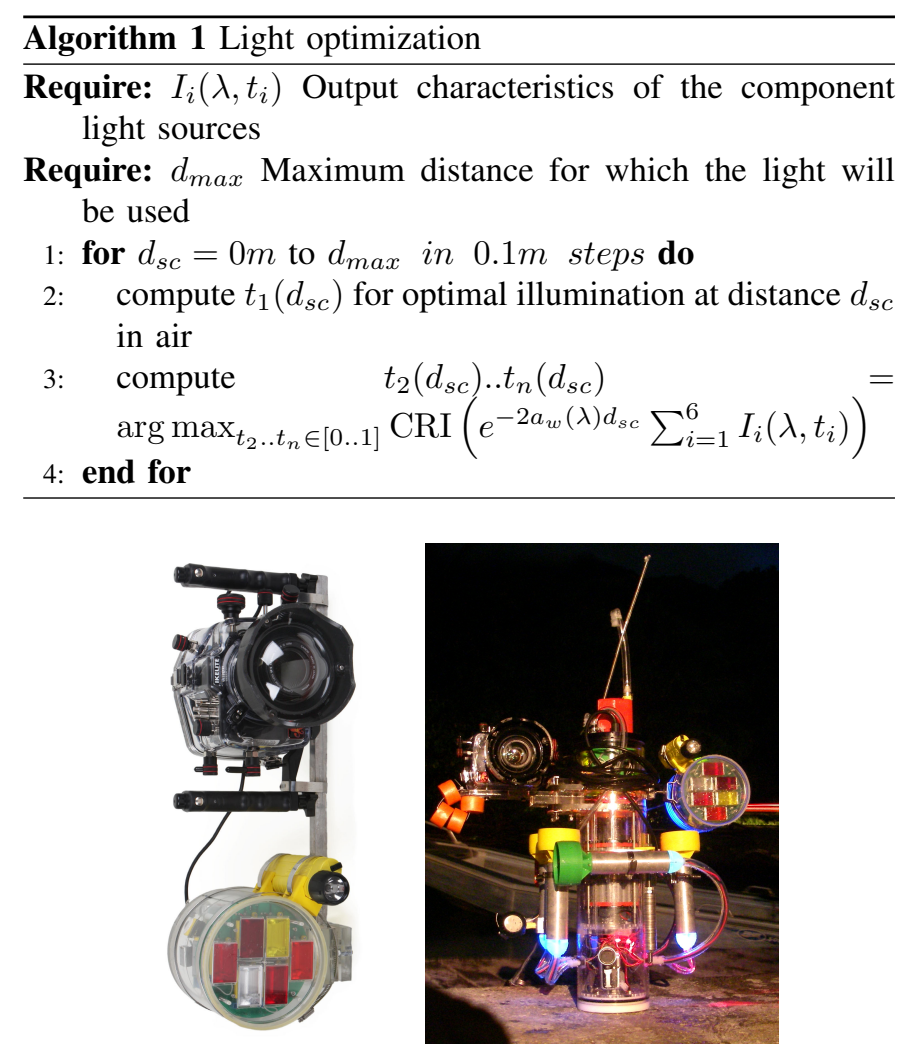

Fig. 7. (Left) The adaptive illumination device consists of six colored flashes with adjustable power. The device is attached to a standard digital camera in an underwater housing. (Right) Imaging apparatus attached to an underwater robot at the end of a mission.

The range of obtainable Color Rendering Index for $\hat{I}(\lambda)$ depends on the choice of the source light components $I_{1}(\lambda) . . I_{n}(\lambda)$. This is restricted by the available light source technologies and filters, and is discussed in Section V. The aim is to obtain a CRI of at least $90 \%$, which is equivalent to the best artificial light sources in air.

\section{IMAGING APPARATUS}

We developed and built a light source prototype capable of adaptive illumination called AquaLight. The light source can be used as an external flash for a digital SLR camera (as seen in Figure 7(Left)), as a free-standing illumination unit, or attached to an underwater robot or sensor node. Figure 7(Right) shows the apparatus attached to an underwater robot.

The light source is composed of 6 independently controlled Xenon strobes. Each strobe is capable of energy discharge up to 50J. The strobes have different but fixed spectra. One is unfiltered and capable of full light spectrum illumination. The other five strobes are filtered with increasingly longer cutoff long pass optical filters. The choice of filters was based on availability and simulation. We optimized for maximum corrected light output for objects at practical underwater photographic range (e.g distances $d_{s c}$ between $0 \mathrm{~m}$ and $5 \mathrm{~m}$ ). The output spectrum of each flash was measured with a calibrated 
radiometric spectrometer. Adaptive illumination is achieved by controlling the strobes' output power, or, equivalently, the energy discharged by each individual flash. Energy discharge is controlled by timing the flash so as to achieve the desired CRI.

More specifically, a Xenon discharge lamp is used as the light source. We chose for a Xenon lamp for its uniform output power across the visible spectrum, which is very similar to sun light. The Xenon lamp is capable of the highest instantaneous output power for its size (thus its widespread use in general photography). One of the flashes is unfiltered and provides the power required for the short wavelengths of the spectrum. Each of the other 5 component light sources has a Xenon discharge lamp and a long-pass filter. Filters are needed to generate different spectra for the 6 light sources. Long-pass filters are used in order to improve the overall efficiency of the device. Since the attenuation in water increases with the wavelength, more power is needed at longer wavelengths than the shorter wavelengths for any distance. The filters were chosen using simulation and maximization of the CRI and illumination power. The cut-off wavelengths for the 5 filters are $475 \mathrm{~nm}, 575 \mathrm{~nm}, 600 \mathrm{~nm}, 600 \mathrm{~nm}$, and 630nm.

For the duration of their discharge, the Xenon lamps are powered by a capacitor bank. Each lamp has an associated $1080 \mu \mathrm{F}$ capacitor, for a maximum discharge energy of 33J. The capacitors are charged to $350 \mathrm{~V}$ by an inverter. The entire unit is powered by a $11.7 \mathrm{~V}, 2.1 \mathrm{Ah}$ Lithium-Polymer battery which provides enough power for approximatively 300 full power flashes.

An NXP LPC2148 CPU together with Xilinx XC2C256 CPLD control the timing of the 6 flashes. The timing is based on the distance to the subject and a precomputed lookup table of distance vs power requirements. In our system, the distance to subject can be input to the unit through a user interface (e.g. distance can be set manually using magnet to set a counter implemented with magnetic switches), camera auto-focus information, or it can be automatically determined using a distance sensor such as an ultrasonic pinger. The data we report in this paper was collected using manually set distance and auto-focus information using an Olympus camera. We found that the ultrasonic pinger (Teledyne/Benthos PSA916) performed poorly for our application (swimming pool conditions as well typical reef environment).

The flash electronics are housed in a water and pressure resistant enclosure made out of clear acrylic. Two underwater connectors are placed on the back of the enclosure. One is used for the electrical connection between the camera and the flash (for synchronization). The other is used for interface with the ultrasonic pinger, programming and battery re-charging. The flash is attached to the camera through a rigid fixture, which ensures they point in the same direction (see Figure 7).

\section{Evaluation}

Adaptive illumination has been evaluated in a series of the pool and ocean experiments. The goal was to measure the color accuracy and to compare the results with existing methods.

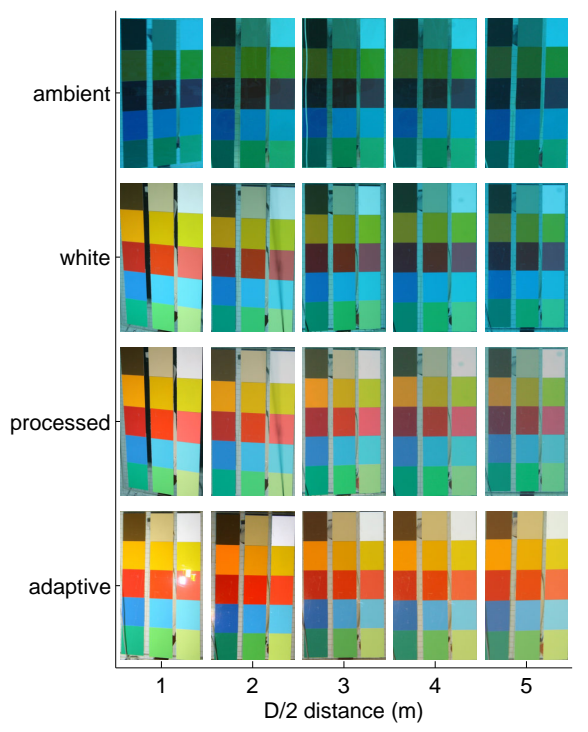

Fig. 8. Results of imaging the color palette in water. The columns correspond to the distance between the light source and the object: $1 \mathrm{~m}, 2 \mathrm{~m}, 3 \mathrm{~m}, 4 \mathrm{~m}$, and $5 \mathrm{~m}$ (note this corresponds to $d_{s c}$ in the main document. The rows show the image obtained using ambient light (first row), white strobe (second row), post-processed white strobe image (third row), and adaptive illumination (fourth row).

We ran the adaptive illumination optimization algorithm for distances between $0.1 \mathrm{~m}$ and $5 \mathrm{~m}$ (in $0.1 \mathrm{~m}$ increments) and determined the optimal power ratios between the flashes. Table I shows the relative power of six flashes for illuminating an object at distance $d_{s c}$ away in water and the resulting $C R I$. We note that the predicted $C R I$ is above $95 \%$ in all cases. The best available artificial light sources are halogen incandescent bulbs which have a CRI of $98 \%$ to $100 \%$, while fluorescent illumination has a CRI of $75 \%$. In Table I F1 is the white flash which was kept at a constant power. Very little power is required from F1 relative to the other flashes. F2 is the yellow flash which contributes to the overall high CRI, but for longer distances a light red flash would have been more effective. The choice of filters was done empirically.

The flash power settings for each distance are stored in the microcontroller's memory of the adaptive illumination device. When taking a picture, given the distance to the desired object, the corresponding power settings are selected from this table.

The experimental methodology consisted of imaging the test object at distance $d_{s c}$ varying between $1 \mathrm{~m}$ and $5 \mathrm{~m}$ (in $1 \mathrm{~m}$ increments) using (1) ambient light, (2) white light strobe, (3) white light strobe and post-processing, and (4) adaptive illumination. The test object was also imaged in air for groundtruth. The test object was a custom made waterproof color palette with 15 colors distributed evenly in the color space (see Figure 8). Distance was determined with a measuring tape and was set manually using the system's magnetic switches. We also used the camera's auto-focus information to automatically 
TABLE I

THE RElATIVE POWER OF THE 6 FLASHES AT DIFFERENT DisTANCES AND THE EXPECTED CRI. NOT THAT THE OBJECT IS AT DISTANCE $\frac{D}{2}$ FROM THE LIGHT SOURCE.

\begin{tabular}{|c|c|c|c|c|c|c|c|}
\hline$\frac{D}{2}$ & $F_{1}$ & $F_{2}$ & $F_{3}$ & $F_{4}$ & $F_{5}$ & $F_{6}$ & $C R I$ \\
\hline $1 \mathrm{~m}$ & 0.0652 & 0.0227 & 0.0405 & 0.0285 & 0.0272 & 0.0188 & 99.26 \\
$2 \mathrm{~m}$ & 0.0652 & 0.0317 & 0.0789 & 0.0710 & 0.0517 & 0.0302 & 98.56 \\
$3 \mathrm{~m}$ & 0.0652 & 0.0421 & 0.1798 & 0.1518 & 0.1425 & 0.1090 & 97.97 \\
$4 \mathrm{~m}$ & 0.0652 & 0.0475 & 1.0000 & 1.0000 & 0.9802 & 0.6584 & 96.16 \\
\hline
\end{tabular}

determine the distance to the imaged object. The standard white flash pictures were post processed with the current state of the art method: equalization using a manually marked white sample patch as reference. This method adjusts the R, G, B channels proportionally to yield the white color on the selected sample.

Color accuracy was measured by computing the color distance between manually selected patches of the color palette and the corresponding patches of the image captured in air. For each pair of color patches we converted the colors to the L*a*b* color space [5] and computed the resulting Euclidean distance. Since the $\mathrm{L}^{*} \mathrm{a} \mathrm{b}^{*}$ color space was specifically designed to preserve the perceptual color distance, the Euclidean distance is an accurate representation of the perceptual color difference. The smaller the distance the better the color accuracy. We used $\mathrm{L}^{*} \mathrm{a}^{*} \mathrm{~b}^{*}$ color space due to the difficulty of measuring CRI experimentally (i.e. procuring the precise color samples used in CRI's definition).

Figure 10 shows the average $L^{*} a^{*} b *$ error for all 15 colors in the palette for each distance. The numerical values for these averages is shown in Table II. The ambient light performs the worst as expected. White flash performs well at $1 \mathrm{~m}$ (and below), but the performance decreases significantly for greater distances. The post processed white flash under-performs adaptive illumination. Its performance decreases steadily with a lower slope than the white flash. Adaptive illumination has no significant decrease in quality up to the measured distance.

Figures 9 show experimental data for four colors from the palette: yellow, red, green and blue. We note that the images taken with the adaptive illumination method have low constant $\mathrm{L} * \mathrm{a} * \mathrm{~b} *$ distances from ground-truth for all distances (accurate colors), while the images taken with the other methods have L*a*b* distances that increase with distance (that is, the rendering is increasingly inaccurate in color).

As expected, not all the colors are affected in the same way. (see Figure 9). Red is most affected by water and it is hardly recoverable by post-processing. Blue is the least affected color. All methods render blue well. Green and the brown - the most commonly occurring natural colors are significantly distorted. This figure also shows distance-dependent $\mathrm{L}^{*} \mathrm{a} * \mathrm{~b} *$ values and the perceived colors the L*a*b* values map to, as computed by ambient light (top row), white flash (second row), postprocessed white flash (third row), and adaptive illumination (fourth row).

An additional suite of pool experiments was done using the camera auto-focus information for automatically setting
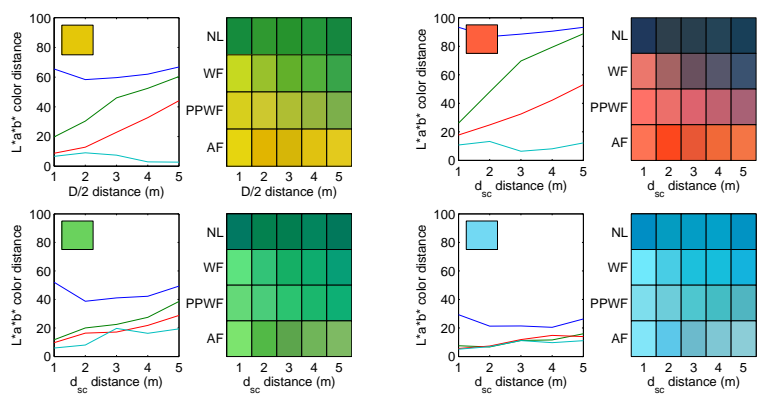

Fig. 9. The $\mathrm{L}^{*} \mathrm{a}^{*} \mathrm{~b} *$ color error (left graphs) and visual appearance (right graphs) for four colors extracted from the color palette (yellow, red, green and blue), as captured by the four imaging methods: ambient light (blue curve), white strobe (green curve), white strobe followed by post-processing (red curve), and adaptive illumination (cyan curve). In the left graphs, the smaller the error, the more accurate the color. The ambient light method performs poorly, the white flash and postprocessed white flash methods degrade with distance, and the adaptive illumination curve remains constant for all distances. The right graphs present the colors extracted from the color palette, as captured by the camera. The columns of the matrix correspond to distance. The rows correspond to the method used (NL for ambient light, WF is white flash, PPWF is postprocessed white flash and AF is adaptive illumination).

the distance to the imaged object. The camera used in these experiments was an Olympus E520 SLR camera. Data from these tests is very similar to the data from the tests where the distance was measured externally.

Underwater imaging experiments have also been conducted in the field at four sites: Fiji, Tahiti, Hawaii, and Grand Cayman. In each experiment images were taken at measured distances using the white flash and the adaptive flash. The images were compared visually. Figure 1 shows typical images using white strobe and adaptive illumination.

We installed the adaptive illumination device on our underwater robot and commanded the robot to swim around a coral head taking a dense sequence of color-accurate photographs. These photographs can be mosaiced to reconstruct the coral head. The robot experiment demonstrated that color-accurate underwater images can be gathered automatically.

\section{CONCLUSIONS}

Adaptive illumination computes the color dissipation at a given distance and compensates for color loss by introducing the correct color mix for that distance into the scene. Perceptual adaptive illumination was tested in a variety of environments and was shown to significantly outperform existing methods for underwater photography with respect to image 


\begin{tabular}{|c|c|c|c|c|c|}
\hline Dist/2 & $1 m$ & $2 m$ & $3 m$ & $4 m$ & $5 m$ \\
\hline ambient & 59.4586 & 52.2793 & 53.8530 & 54.2241 & 58.6122 \\
white & 14.9747 & 26.4679 & 37.4749 & 43.9231 & 51.0177 \\
white post-processed & 9.2605 & 14.3705 & 18.9246 & 27.9815 & 34.8542 \\
adaptive & 8.3305 & 11.0851 & 11.3560 & 8.7389 & 9.9966 \\
\hline
\end{tabular}

TABLE II

THE L*A*B* VALUE AVERAGED OVER 15 COLORS.

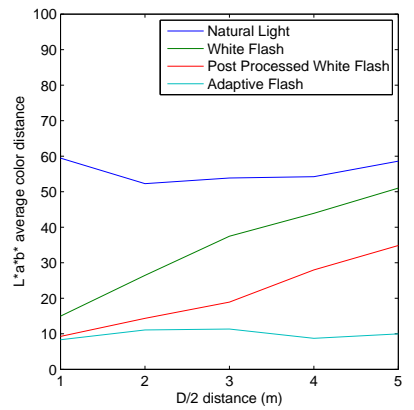

Fig. 10. $\mathrm{L}^{*} \mathrm{a}^{*} \mathrm{~b}^{*}$ average values for 15 colors at $1 \mathrm{~m}, 2 \mathrm{~m}, 3 \mathrm{~m}, 4 \mathrm{~m}$, and $5 \mathrm{~m}$, as computed by 4 different imaging methods: ambient light (blue curve), white strobe (green curve), white strobe with post-processing (red curve), and adaptive illumination (cyan curve).

color accuracy using the $\mathrm{L}^{*} \mathrm{a} * \mathrm{~b} *$ metric space for measuring colors. Color encodes important information about underwater environments and habitats. This work enables the capture of color-accurate underwater images and thus opens the door to automating underwater monitoring and surveillance operations based on color.

Our work demonstrates that color-adaptive illumination is effective for color-accurate imaging in water for objects at a specified or computed distance. Our experimental data provides support for this claim for imaging objects that are up to $5 \mathrm{~m}$ away from the camera. Current methods are cumbersome and do not produce accurate colors at distances greater than $2 \mathrm{~m}$. Our imaging instrument was prototyped in our lab using inexpensive components to demonstrate the concept. The instrument was not optimized for distance. Imaging at distances greater than $5 \mathrm{~m}$ is achievable if the instrument uses more powerful light sources.

Perceptual adaptive illumination renders color accurately for all the objects at the given distance $d$ in the image. Objects further away or closer to the camera will not be rendered correctly because adaptive illumination is distance-dependent. We are currently extending perceptual adaptive illumination from color-accuracy in one image plane to color-accuracy in multiple image planes. We are also working on extensions from still images to video.

Our current work includes field experiments to mosaic underwater scenes using robots equipped with the perceptual adaptive illumination device described in this paper.

\section{REFERENCES}

[1] D. Bellwood. The 'lost' language of fishes. http://www.coralcoe.org.au/news_stories/fishcolour.html.

[2] A.C. Crook. Colour patterns in a coral reef fish: Is background complexity important? J. of eperimental marine biology and ecology, 217(2), 1997.

[3] Martin Edge. The underwater photographer, third edition: Digital and traditional techniques (paperback), 2006

[4] Commission internationale de l'éclairage. Cie $19312^{\circ}$ standard observer, 1931.

[5] Commission internationale de l'éclairage. Supplement no. 2 to cie publication no. 15, colorimetry, 1976.

[6] Jules Jaffe, Kad Moore, John McLean, and Michael Strand. Underwater optical imaging: Status and prospects. Oceanography, 14, 2007.

[7] V. Messmer, G.P. Jones, L. van Herwerden, and P.L. Munday. Genetic and ecological characterisation of colour dimorphism in a coral reef fish. Evironmental Biology of Fishes, 74, 2005.

[8] Hanumant Singh, Roy Armstrong, Fernando Gilbes, Ryan Eustice, Chris Roman, Oscar Pizarro, and Juan Torres. Imaging coral i: Imaging coral habitats with the seabed auv. Subsurface Sensing Technologies and Applications, 5, 2004.

[9] R. C. Smith and K. S. Baker. Optical properties of the clearest natural waters $(200-800 \mathrm{~nm})$. Appl. Opt., 20:177-184, 1981.

[10] A. Stockman, D.I.A. MacLeod, and N.E. Johnson. Spectral sensitivities of human cones. Journal of the Optical Society of America, 10:2491$2521,1993$.

[11] L.A. Torres-Mndez and G. Dudek. A statistical learning-based method for color correction of underwater images. Research on Computer Science, 17, 2005.

[12] Iuliu Vasilescu, Carrick Detweiler, Marek Doniec, Daniel Gurdan, Steffan Sosnowski, Jan Stumpf, , and Daniela Rus. Amour v: a hovering energy efficient underwater robot capable of dynamic payloads. The International Journal of Robotics Research, 29(5):547-570, 2010.

[13] Stefan B. Williams, Oscar Pizarro, Martin How, Duncan Mercer, George Powell, Justin Marshall, and Roger Hanlon. Surveying noctural cuttlefish camouflage behaviour using an auv. In ICRA, pages 214-219, 2009.

[14] G. Wyszecki. Proposal for a new color-difference formula. JOSA, 53:1318-1319, 1963.

[15] A. Yamashita, M. Fujii, and T. Kaneko. Color registration of underwater images for underwater sensing with consideration of light attenuation. Robotics and Automation, 2007 IEEE International Conference on, pages 4570-4575, April 2007. 